\title{
Denoising Electron-energy Loss Data Using Non-local Means Filters
}

\author{
Niklas Mevenkamp ${ }^{1}$, Benjamin Berkels ${ }^{1}$ and Martial Duchamp ${ }^{2}$ \\ 1. AICES Graduate School, RWTH Aachen University, Aachen, Germany \\ 2. School of Materials Science and Engineering, Nanyang Technological University, Singapore
}

The acquisition of high resolution electron-energy loss (EEL) datasets in a transmission electron microscope (TEM) is still challenging as the total dose and the electron rate are limited by the sensibility of the specimen to electron radiation. Thus, the experimentalists have to cope with noisy spectroscopic datasets and apply post-processing techniques to extract the most information.

Various multivariant analysis (MVA) methods have been used to denoise [1] or extract physically interpretable information [2-4]. The idea is to project the dataset on a subspace of lower dimension, which, in the ideal case, is described by physically meaningful spectra. MVA methods assume that any spectrum is a linear combination of a given set of spectra but does not account for the redundant information in neighbouring pixels. Indeed, MVA techniques look for spectral rather than spatial similarities.

On the other hand, local averaging technique, look for similarity in the spatial dimensions. Block matching methods based on the non-local means filter (NLM) have been applied to improve the signal to noise of scanning (S-)TEM images [5]. Given that objects of interest within the image actually occur multiple times, this approach has proven to offer a truthful reconstruction while sacrificing much less spatial resolution than with local averaging. Based on the success of this technique to STEM images, we apply a similar procedure to EELS data. The search of matching spectra and the following averaging are presented Fig. 1.

We applied the NLM method on a EELS dataset recorded from a defective amorphous silicon solar cell previously analysed by MVA [6]. We focused the study at the Zn K and Si K edges due to the lower signal to noise ratio at higher energy losses. NLM and principal component analysis (PCA) maps have an improved signal to noise ratio compare to raw maps (Figs. 2a,b). Extra features appears in the PCA Zn map compare to the raw and NLM maps (marked by white circles in the Fig. 2a) due to the limited dimension of the sub-space used to reconstruct the PCA dataset. A second unwanted effect found in the PCA maps is a reduction of the spatial resolution compared to the raw and NLM maps (Fig. 2b). Figure $2 \mathrm{c}$ shows specific spectra extracted from the same location for the raw, NLM and PCA dataset. Even though the number of components for the PCA decomposition was kept low to get a larger signal to noise ratio, NLM spectra are less noisy.

The application of NLM to EEL datasets is shown to better recover the ground truth information while keeping the original spatial resolution. A further application of this technique to atomic resolution spectroscopic datasets promises to overcome the limitations encountered while analysing noisy spectroscopic datasets of beam sensitive specimen. Analysis of atomically resolved EEL and EDX datasets are ongoing and will be the subject of further reports.

References: 
[1] P. Trebbia and N. Bonnet, Ultramicroscopy 34 (1990), p. 165.

[2] N. Bonnet and D. Nuzillard, Ultramicroscopy 102 (2005), p. 327.

[3] N. Dobigeon and N. Brun Ultramicroscopy 120 (2012), p. 25.

[4] P. Burdet et al, Acta Mater. 61 (2013), p. 3090.

[5] N. Mevenkamp et al, Adv. Struct. Chem. Imaging 1 (2015), p. 3.

[6] M. Duchamp et al, Appl. Phys. Lett. 102 (2013), p. 133902.

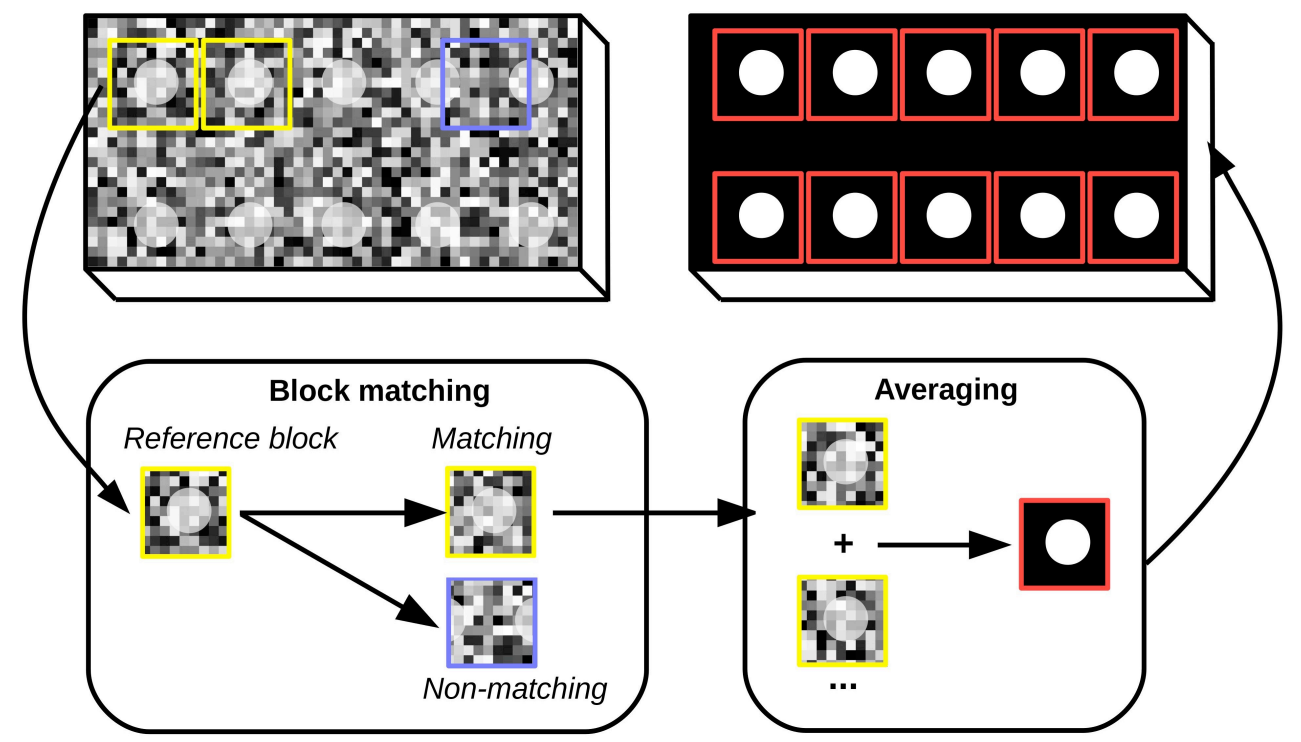

Figure 1. Illustration of the NLM denoising procedure for one selected reference block. The reference block is shifted pixel by pixel and line by line across the entire scan area. Here, the search for similar blocks takes into account the entire spectrum in the block at once.
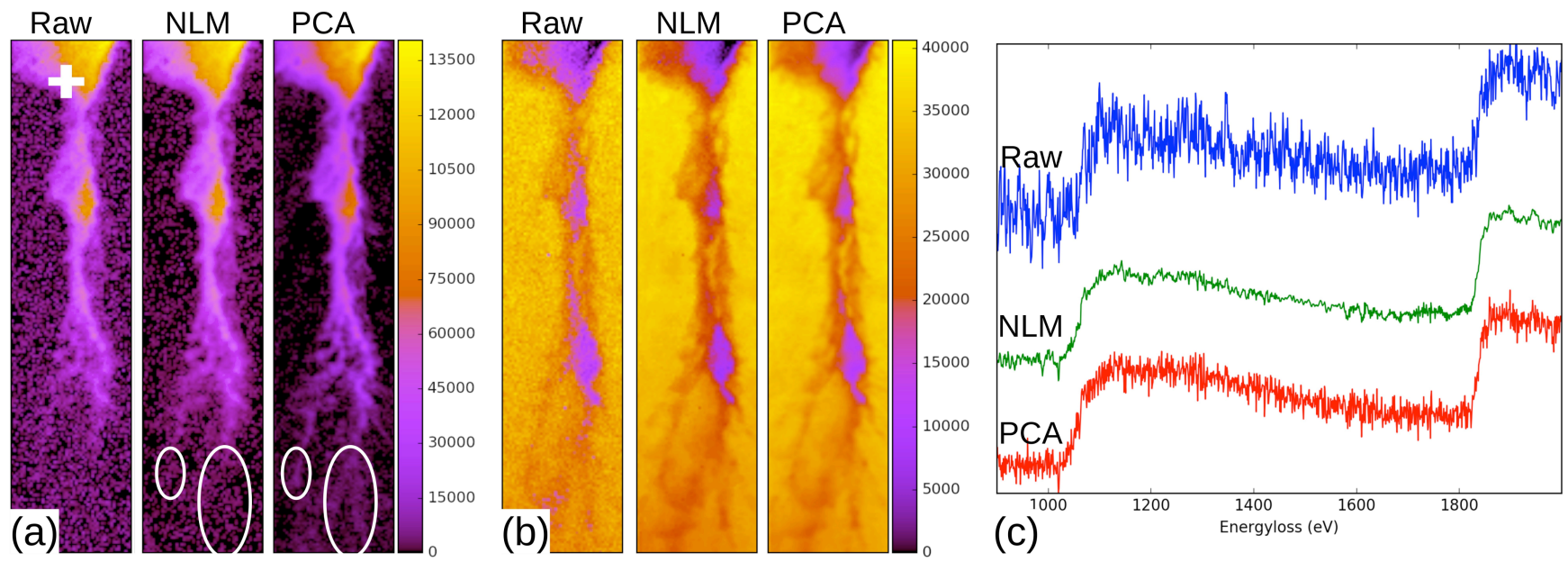

Figure 2. EEL maps of a defective region in the region of the solar cell at the (a) $\mathrm{Zn} \mathrm{K}$ and (b) Si K edges. From left to right, maps integrated from the raw dataset, after applying our NLM denoising procedure and after applying PCA. (c) Three background-subtracted EEL spectra from the raw dataset, after applying our NLM denoising procedure and after applying PCA. The white arrow in (a) shows the pixel from which the spectra where extracted. The circles in (a) are explained in the text. The field of view in (a-b) is $0.31 \mu \mathrm{m}$ by $1.34 \mu \mathrm{m}$. 